\title{
Effects of Simultaneous Atrioventricular Pacing on Atrial Refractoriness and Atrial Fibrillation Inducibility: Role of Atrial Mechanoelectrical Feedback
}

\author{
HUNG-FAT TSE, M.D., FRANK PELOSI, M.D., HAKAN ORAL, M.D., \\ BRADLEY P. KNIGHT, M.D., S. ADAM STRICKBERGER, M.D., \\ and FRED MORADY, M.D. \\ From the Division of Cardiology, Department of Internal Medicine, \\ University of Michigan Medical Center, Ann Arbor, Michigan
}

\begin{abstract}
Atrial Mechanoelectrical Feedback. Introduction: The purpose of this study was to evaluate the effects of an acute increase in atrial pressure on refractoriness (mechanoelectrical feedback) and the vulnerability to atrial fibrillation (AF) and to investigate the effects of autonomic blockade and verapamil on mechanoelectrical feedback in humans.

Methods and Results: Right atrial pressure and effective refractory period (ERP) at the right atrial appendage (RAA) and high right atrial septum were measured during sinus rhythm, and during atrial and simultaneous AV pacing at a cycle length of 300 msec, either in the absence $(n=25)$ or presence $(n=22)$ of pharmacologic autonomic blockade. In another 15 patients, the protocol was performed before and after infusion of verapamil $0.15 \mathrm{mg} / \mathrm{kg}$. In the absence of autonomic blockade, AV pacing resulted in a higher mean right atrial pressure $(11.7 \pm 3.3$ vs $4.3 \pm 3.0 \mathrm{mmHg}, \mathrm{P}<0.001)$ and a shorter atrial RAA ERP $(144 \pm 23 \mathrm{msec}$ vs $161 \pm 21 \mathrm{msec} ; \mathrm{P}<0.001)$ compared with atrial pacing; AF was induced more often during AV pacing $(87 \%)$ than during atrial pacing $(20 \%)$ and was related directly to the right atrial pressure $(r=0.39, P=0.004)$ and indirectly to the RAA ERP $(r=-0.42, P<0.001)$. The susceptibility to sustained AF was greatly enhanced by autonomic blockade. Verapamil markedly attenuated the shortening of ERP and the propensity for AF that occurred during simultaneous AV pacing.

Conclusion: An acute increase in atrial pressure during tachycardia is associated with shortening of atrial refractoriness and a propensity for AF, i.e., atrial mechanoelectrical feedback, which may be enhanced by autonomic blockade and attenuated by calcium channel blockade. ( $J$ Cardiovasc Electrophysiol, Vol. 12, pp. 43-50, January 2001)
\end{abstract}

atrial fibrillation, atrial refractory period, mechanoelectrical feedback

\section{Introduction}

An increase in atrial pressure predisposes to the development of atrial fibrillation (AF), ${ }^{1-3}$ and the arrhythmogenic effects of an increase in pressure may be mediated by the phenomenon of mechanoelectrical feedback, in which atrial refractoriness changes in response to mechanical stretch.4-6 Recent experimental studies demonstrated that mechanical stretch of atrial myocardium results in a shortening of atrial refractoriness and an increase in vulnerability to AF. ${ }^{6,7}$ However,

Address for correspondence: Fred Morady, M.D., Division of Cardiology, B1F245, University of Michigan Medical Center, 1500 East Medical Center Drive, Ann Arbor, MI 48109-0022. Fax: 734-9367026.

Manuscript received 21 August 2000; Accepted for publication 2 October 2000. prior studies in humans observed inconsistent effects of atrial stretch on atrial refractoriness, ${ }^{8-12}$ and arrhythmogenic effects have not been described. The goal of the present study was to clarify whether atrial mechanoelectrical feedback occurs in humans, and whether it contributes to the generation or maintenance of AF.

\section{Methods}

\section{Patient Characteristics}

The subjects of this study were 62 patients who underwent radiofrequency catheter ablation of paroxysmal supraventricular tachycardia. Exclusion criteria consisted of a baseline rhythm of AF or atrial flutter, the presence of structural heart disease, the inability to achieve a stable electrode catheter position in the right atrium throughout the study, or sustained AF during the study protocol requiring electrical cardioversion on more than 
two occasions. One of these exclusion criteria was present in 14 of 76 patients who were considered for inclusion in this study. The 62 remaining subjects consisted of 32 men and 30 women (mean age \pm SD: $46 \pm$ 14 years). Mean left ventricular ejection fraction was $0.60 \pm 0.06$, and mean left atrial diameter by echocardiography was $3.4 \pm 0.4 \mathrm{~cm}$.

\section{Electrophysiologic Procedures}

Electrophysiologic procedures were performed in the fasting state after informed consent was obtained. All antiarrhythmic drug therapy was discontinued at least five half-lives before the procedure. Three quadripolar electrode catheters were inserted into a femoral vein and initially positioned in the high right atrium, His-bundle position, and right ventricular apex. Midazolam was used for conscious sedation. Leads $\mathrm{V}_{1}$, I, II, and III and the intracardiac electrograms were recorded on paper or optical disk. Pacing was performed with a programmable stimulator (Bloom Associates, Ltd., Reading, PA, USA) using stimuli that had a duration of $2 \mathrm{msec}$.

\section{Study Protocol}

The study protocol was approved by the Human Research Committee and was performed upon completion of the clinically indicated portion of the electrophysiologic procedure. A quadripolar electrode catheter was positioned in the right atrial appendage (RAA) or at the high right atrial septum (AS), such that the pacing threshold was $<1 \mathrm{~mA}$ (mean $0.9 \pm 0.3$ ). Another electrode catheter was positioned at the right ventricular apex for ventricular pacing. Bipolar pacing was performed at a current strength equal to three times the stimulation threshold to ensure stable capture during rapid pacing. The tip of an 8-French, 65-cm sheath inserted in a femoral vein was positioned in the right atrium to measure atrial pressure. Blood pressure was measured with an automatic brachial blood pressure cuff. Mean right atrial pressure and mean blood pressure were recorded during each measurement of atrial effective refractory period (ERP).

Atrial ERP was measured during sinus rhythm by introducing an atrial extrastimulus after every eighth sinus beat at an initial coupling interval of $150 \mathrm{msec}$ and increasing the coupling interval in increments of $5 \mathrm{msec}$ until there was atrial capture. Atrial ERP was defined as the longest S1S2 coupling interval that failed to result in atrial capture.

Atrial ERP then was measured during atrial and simultaneous AV pacing at a cycle length of $300 \mathrm{msec}$, in random order. Simultaneous AV pacing at a cycle length of $300 \mathrm{msec}$ was used to evoke an acute increase in right atrial pressure, ${ }^{8-10}$ and right atrial pacing at the same cycle length was used to control for the effect of an increase in rate on atrial ERP. After 3 minutes of continuous pacing at a cycle length of $300 \mathrm{msec}$ to achieve steady-state conditions, an atrial extrastimulus was introduced at a coupling interval of $100 \mathrm{msec}$. After every eighth drive train stimulus, the coupling interval of the extrastimulus was increased by $5 \mathrm{msec}$, with no pause in the drive train, until the extrastimulus that resulted in atrial capture occurred twice in succession. Atrial and simultaneous AV pacing were performed in a random order, as was the pacing site within the right atrium.

To determine the effects of autonomic blockade, the pacing protocol at the RAA and AS were performed in the initial 25 consecutive patients in the absence of autonomic blockade and in the next 22 consecutive patients after pharmacologic autonomic blockade. Autonomic blockade was achieved by the simultaneous infusion of atropine $0.04 \mathrm{mg} / \mathrm{kg}$ and propranolol $0.2 \mathrm{mg} / \mathrm{kg}$ administered over 5 minutes. ${ }^{13}$ Mean patient weight was $73 \pm 15 \mathrm{~kg}$, and mean atropine and propranolol doses were $2.5 \pm 0.3 \mathrm{mg}$ and $13.2 \pm 2.2 \mathrm{mg}$, respectively.

In another 15 consecutive patients, the effects of verapamil were determined. The pacing protocol at the RAA was performed before and after infusion of verapamil $0.15 \mathrm{mg} / \mathrm{kg}$ over 3 minutes $^{14}$ after pharmacologic autonomic blockade. Mean verapamil dose was $11.7 \pm$ $0.2 \mathrm{mg}$.

Difference in atrial refractoriness $(\triangle E R P)$ was defined as the difference between the ERPs at the RAA and AS. Susceptibility to AF was assessed by noting whether AF was induced by the extrastimulus that resulted in atrial capture during measurement of the atrial ERP at the RAA. Episodes of AF had to be $>3$ seconds in duration to be counted as an episode of AF. Pacing was stopped whenever AF occurred. If the AF lasted $>10$ minutes, electrical cardioversion was performed to restore sinus rhythm. Because short episodes of AF can affect atrial refractoriness for several minutes, ${ }^{15}$ the study protocol was suspended for 10 minutes after spontaneous or electrical cardioversion. To confirm a stable catheter position, the atrial pacing threshold was remeasured before every measurement of atrial ERP and after each electrical cardioversion.

\section{Statistical Analysis}

Continuous variables are expressed as mean $\pm 1 \mathrm{SD}$. Statistical comparisons were performed with Student's $t$-test or by Chi-square analysis, as appropriate. Analysis of variance was used for multiple group comparisons, followed by a Bonferroni-corrected $t$-test. Logistic regression analysis was performed to calculate the inducibility of AF by single atrial premature stimuli during atrial pacing and during simultaneous AV pacing at different atrial pressures and atrial ERPs. $\mathrm{P}<0.05$ was considered statistically significant.

\section{Results}

\section{Hemodynamic Changes}

During sinus rhythm, mean right atrial pressures in the absence of autonomic blockade, in the presence of autonomic blockade, and after infusion of verapamil did not differ significantly (Table 1). During atrial pacing at 
TABLE 1

Hemodynamic Changes

\begin{tabular}{|c|c|c|c|c|}
\hline & $\begin{array}{c}\text { Absence of } \\
\text { Autonomic } \\
\text { Blockade } \\
(\mathbf{n}=25)\end{array}$ & $\begin{array}{c}\text { Presence of } \\
\text { Autonomic } \\
\text { Blockade } \\
(\mathbf{n}=\mathbf{2 2})\end{array}$ & $\begin{array}{c}\text { Verapamil } \\
\text { Infusion } \\
(\mathbf{n}=\mathbf{1 5})\end{array}$ & P Value \\
\hline \multicolumn{5}{|c|}{ Mean AV interval (msec) } \\
\hline Sinus rhythm & $156 \pm 54$ & $170 \pm 35$ & $184 \pm 24$ & $>0.05$ \\
\hline Atrial pacing & $172 \pm 54$ & $213 \pm 36$ & $226 \pm 40$ & $<0.05$ \\
\hline \multicolumn{5}{|c|}{ Mean RAP (mmHg) } \\
\hline Sinus rhythm & $3.6 \pm 2.8^{*}$ & $2.7 \pm 3.5^{*}$ & $3.2 \pm 2.8^{*}$ & $>0.05$ \\
\hline Atrial pacing & $4.3 \pm 3.0^{*}$ & $3.9 \pm 1.7 *$ & $4.3 \pm 2.9^{*}$ & $>0.05$ \\
\hline AV pacing & $11.7 \pm 3.3$ & $11.3 \pm 4.4$ & $13 \pm 2.8$ & $>0.05$ \\
\hline \multicolumn{5}{|c|}{ Changes in mean RAP $(\mathrm{mmHg}) \dagger$} \\
\hline Atrial pacing & $0.7 \pm 2.3^{*}$ & $1.3 \pm 1.8^{*}$ & $1.1 \pm 1.3^{*}$ & $>0.05$ \\
\hline AV pacing & $8.1 \pm 2.9$ & $7.5 \pm 2.8$ & $9.8 \pm 3.4$ & $>0.05$ \\
\hline \multicolumn{5}{|c|}{ Mean BP (mmHg) } \\
\hline Sinus rhythm & $86 \pm 7 *$ & $80 \pm 7$ & $85 \pm 11$ & $>0.05$ \\
\hline Atrial pacing & $84 \pm 9 *$ & $83 \pm 15$ & $81 \pm 17$ & $>0.05$ \\
\hline AV pacing & $72 \pm 16$ & $79 \pm 19$ & $80 \pm 12$ & $>0.05$ \\
\hline \multicolumn{5}{|c|}{ Changes in mean $\mathrm{BP}(\mathrm{mmHg}) \dagger$} \\
\hline Atrial pacing & $-1 \pm 10^{*}$ & $-0.5 \pm 20$ & $-4.4 \pm 14$ & $>0.05$ \\
\hline AV pacing & $-14 \pm 17$ & $-3 \pm 14$ & $-5.5 \pm 14$ & 0.02 \\
\hline
\end{tabular}

Variables are expressed as mean $\pm 1 \mathrm{SD}$.

$* \mathrm{P}<0.05$ compared with AV pacing.

$\dagger$ Changes in pressure as compared with sinus rhythm.

$\mathrm{BP}=$ blood pressure; $\mathrm{RAP}=$ right atrial pressure .

a cycle length of $300 \mathrm{msec}$, the mean AV interval in the absence of autonomic blockade was significantly shorter than those in the presence of autonomic blockade, and after infusion of verapamil, but did not differ significantly compared to during sinus rhythm (Table 1). Ten patients $(16 \%)$ developed 2:1 AV conduction during atrial pacing. However, mean right atrial pressures in the absence of autonomic blockade, in the presence of autonomic blockade, and after infusion of verapamil did not differ significantly and remained unchanged compared to during sinus rhythm (Table 1).

During simultaneous AV pacing at a cycle length of $300 \mathrm{msec}$, mean right atrial pressure increased significantly compared to during sinus rhythm and atrial pacing, and increased to a similar degree in the absence of autonomic blockade, in the presence of autonomic blockade, and after infusion of verapamil (Table 1).

During sinus rhythm, there were no significant differences in mean blood pressure in the absence of autonomic blockade, in the presence of autonomic blockade, or after infusion of verapamil (Table 1). In the absence of autonomic blockade, mean blood pressure decreased during simultaneous AV pacing, but not during atrial pacing (Table 1). In the presence of autonomic blockade, regardless of whether or not verapamil had been infused, there were no significant changes in mean blood pressure during atrial or simultaneous AV pacing as compared to during sinus rhythm (Table 1).

\section{Atrial Refractoriness}

\section{No autonomic blockade}

Mean sinus cycle length was $851 \pm 151 \mathrm{msec}$, and mean atrial ERPs at the RAA and AS were $224 \pm 30$ msec and $239 \pm 24 \mathrm{msec}$, respectively. Compared to during sinus rhythm, the mean RAA ERP shortened significantly to $161 \pm 21 \mathrm{msec}$ during atrial pacing $(\mathrm{P}<$ 0.001). There was a further shortening of the ERP at the RAA to $144 \pm 23 \mathrm{msec}$ during simultaneous AV pacing $(\mathrm{P}=0.003$; Table 2). ERP at the AS decreased significantly and to the same extent as at the RAA during atrial and simultaneous AV pacing (Table 2).

As compared with atrial ERPs at the RAA, atrial ERPs at the AS were significantly longer during sinus rhythm and during simultaneous AV pacing, but not during atrial pacing (Fig. 1). $\triangle \mathrm{ERP}$ was not significantly greater during simultaneous AV pacing than during atrial pacing or sinus rhythm $(\mathrm{P}=0.2$; Table 2$)$.

\section{Autonomic blockade}

Mean sinus cycle length after autonomic blockade was $682 \pm 77 \mathrm{msec}$. Mean atrial ERPs at the RAA and AS were $216 \pm 12 \mathrm{msec}$ and $233 \pm 17 \mathrm{msec}$, respectively. Compared to during sinus rhythm, mean RAA ERP shortened significantly to $170 \pm 19 \mathrm{msec}$ during atrial pacing $(\mathrm{P}<0.001)$, with a further shortening to $146 \pm 17 \mathrm{mmHg}$ during simultaneous AV pacing $(\mathrm{P}=$ 0.01; Table 2). ERP at the AS decreased significantly and to the same extent as at the RAA during atrial and simultaneous AV pacing (Table 2).

As compared with atrial ERPs at the RAA, atrial ERPs at the AS were significantly longer during sinus rhythm and during simultaneous AV pacing, but not during atrial pacing (Fig. 1). $\triangle \mathrm{ERP}$ was significantly greater during simultaneous $\mathrm{AV}$ than during sinus rhythm and/or atrial pacing $(\mathrm{P}=0.01$; Table 2$)$. 
TABLE 2

Atrial Refractory Periods and Prevalence of Induced Atrial Fibrillatoin in the Absence and the Presence of Autonomic Blockade

\begin{tabular}{|c|c|c|c|}
\hline & $\begin{array}{c}\text { Absence of } \\
\text { Autonomic } \\
\text { Blockade }(n=25)\end{array}$ & $\begin{array}{c}\text { Presence of } \\
\text { Autonomic } \\
\text { Blockade }(n=22)\end{array}$ & P Value \\
\hline Mean sinus cycle length (msec) & $851 \pm 152$ & $682 \pm 77$ & 0.02 \\
\hline \multicolumn{4}{|l|}{ RAA ERP (msec) } \\
\hline Sinus rhythm & $224 \pm 30 * 末$ & $216 \pm 12^{*}+$ & 0.4 \\
\hline Atrial pacing & $161 \pm 21 *$ & $170 \pm 19 *$ & 0.3 \\
\hline AV pacing & $144 \pm 23$ & $146 \pm 17$ & 0.6 \\
\hline \multicolumn{4}{|l|}{ Changes in RAA ERP (msec) $\dagger$} \\
\hline Atrial pacing & $-62 \pm 28^{*}$ & $-46 \pm 16^{*}$ & 0.1 \\
\hline AV pacing & $-80 \pm 38$ & $-70 \pm 15$ & 0.9 \\
\hline \multicolumn{4}{|l|}{ AS ERP (msec) } \\
\hline Sinus rhythm & $239 \pm 20^{*}+$ & $233 \pm 17 *+$ & 0.4 \\
\hline Atrial pacing & $171 \pm 13$ & $181 \pm 13$ & 0.08 \\
\hline AV pacing & $164 \pm 18$ & $182 \pm 16$ & 0.03 \\
\hline \multicolumn{4}{|l|}{ Changes in AS ERP $(\mathrm{msec}) \dagger$} \\
\hline Atrial pacing & $-67 \pm 28$ & $-52 \pm 24$ & 0.1 \\
\hline AV pacing & $-75 \pm 30$ & $-51 \pm 27$ & 0.03 \\
\hline \multicolumn{4}{|l|}{$\Delta \mathrm{ERP}(\mathrm{msec})$} \\
\hline Sinus rhythm & $15 \pm 22$ & $17 \pm 26^{*}$ & 0.8 \\
\hline Atrial pacing & $10 \pm 18$ & $11 \pm 25^{*}$ & 0.9 \\
\hline AV pacing & $20 \pm 26$ & $36 \pm 16$ & 0.04 \\
\hline \multicolumn{4}{|l|}{ Atrial fibrillation } \\
\hline Sinus rhythm & $0(0 \%)^{*}$ & $0(0 \%)^{*}$ & 1.0 \\
\hline Atrial pacing & $5 / 25(20 \%)^{*}$ & $4 / 22(18 \%)^{*}$ & 1.0 \\
\hline AV pacing & $22 / 25(87 \%)$ & 20/22 (91\%) & 1.0 \\
\hline \multicolumn{4}{|l|}{ Atrial fibrillation, duration $>10 \mathrm{~min}$} \\
\hline Sinus rhythm & $0(0 \%)$ & $0(0 \%)^{*}$ & 1.0 \\
\hline Atrial pacing & $0(0 \%)$ & $0(0 \%)^{*}$ & 1.0 \\
\hline AV pacing & $2 / 22(9 \%)$ & $10 / 20(50 \%)$ & 0.006 \\
\hline
\end{tabular}

Variables are expressed as mean $\pm 1 \mathrm{SD}$.

$* \mathrm{P}<0.05$ compared with AV pacing; $\$ \mathrm{P}<0.05$ compared with atrial pacing.

$\dagger$ Changes in pressure as compared with sinus rhythm.

$\mathrm{AS}=$ atrial septum; ERP $=$ effective refractory period; RAA $=$ right atrial appendage.

\section{Absence versus presence of autonomic blockade}

Autonomic blockade had no significant effect on ERP at the RAA or AS during sinus rhythm (Table 2). Autonomic blockade also did not affect the response of RAA ERP to atrial or simultaneous AV pacing (Table 2). However, there was less shortening of ERP at the AS in the presence than in the absence of autonomic blockade during simultaneous AV pacing (Table 2). This resulted in a $\triangle E R P$ that was greater in the presence than in the absence of autonomic blockade during simultaneous AV pacing (Table 2).

\section{Effect of verapamil}

Atrial ERPs measured immediately before administration of verapamil are listed in Table 3. After administration of verapamil, mean ERP at the RAA during sinus rhythm was $231 \pm 24 \mathrm{msec}$. Mean RAA ERP decreased significantly during atrial pacing, with no further significant change during simultaneous AV pacing (Table 3 and Fig. 2). Verapamil significantly attenuated the shortening of atrial ERP that occurred in response to atrial and simultaneous AV pacing (Fig. 2).

\section{Atrial Vulnerability}

\section{Absence and presence of autonomic blockade}

In the setting of sinus rhythm, AF never was induced during measurement of ERPs, either in the absence or presence of autonomic blockade. In the setting of atrial pacing at a cycle length of $300 \mathrm{msec}$, AF occurred during measurement of atrial ERP in the absence and presence of autonomic blockade in $20 \%$ and $18 \%$ of patients, respectively.

During simultaneous AV pacing at a cycle length of $300 \mathrm{msec}$, the prevalence of AF during measurement of atrial ERP increased significantly to $87 \%$ and $92 \%$ of patients, in the absence and the presence of autonomic blockade, respectively (Table 2).

Both in the absence and the presence of autonomic blockade, the inducibility of AF during determination of atrial ERP correlated with mean right atrial pressure (Fig. 3) and was inversely related to atrial ERP (Fig. 4), but not with $\triangle \mathrm{ERP}(\mathrm{r}=0.2$ and 0.3 , respectively; $\mathrm{P}=0.5$ ).

Autonomic blockade did not influence the overall prevalence of induced AF during sinus rhythm, atrial pacing, or simultaneous AV pacing (Table 2). However, the prevalence of AF that lasted at least 10 minutes induced during simultaneous AV pacing was signifi- 

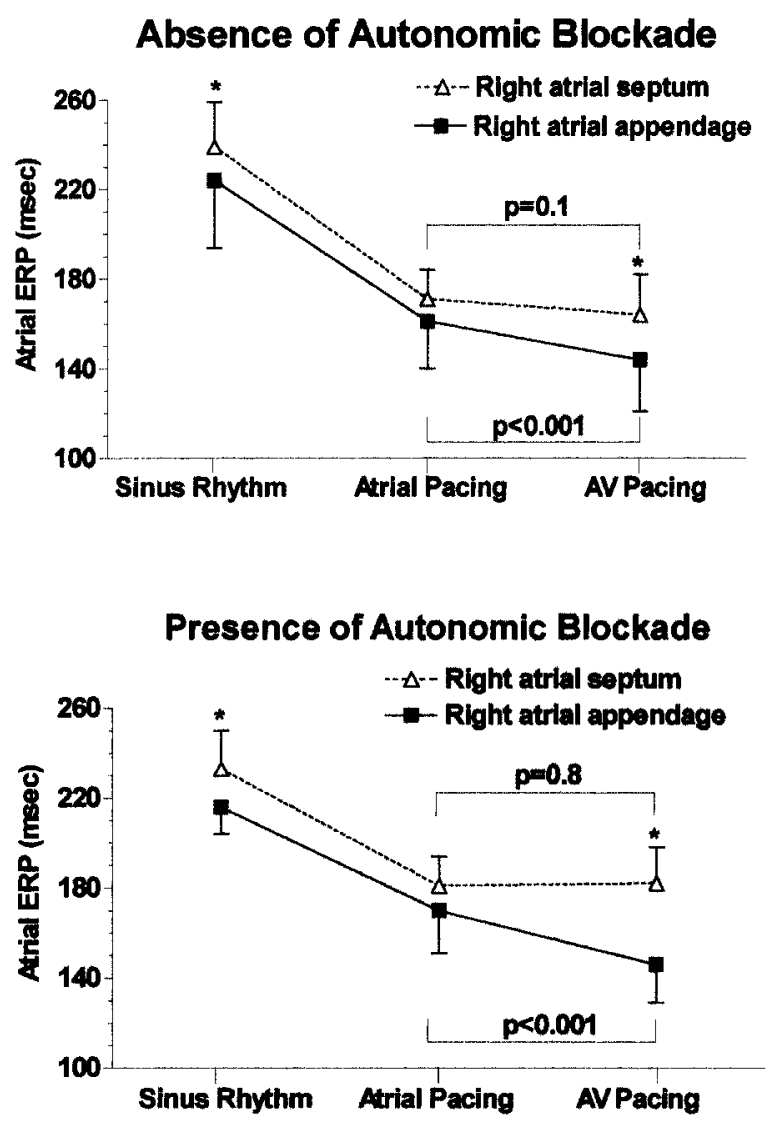

Figure 1. Atrial effective refractory period (ERP) measured during sinus rhythm, during atrial pacing at a cycle length of $300 \mathrm{msec}$, and during $A V$ pacing at the right atrial appendage (ם) and at the right atrial septum $(\Delta)$ in the absence (top panel) or presence (bottom panel) of autonomic blockade. Mean values $\pm 1 S D$ are shown. $* P<0.05$ compared with sinus rhythm; $* * P<0.05$ compared with $A V$ pacing.

cantly higher in the presence than in the absence of autonomic blockade (Table 2). Logistic regression analysis demonstrated that only $\triangle \mathrm{ERP}$ was correlated with the persistence of induced $\mathrm{AF}>10$ minutes $(\mathrm{r}=0.4, \mathrm{P}=$ $0.03)$, but not with atrial ERP $(r=0.3, P=0.2)$ or mean right atrial pressure $(\mathrm{r}=0.2, \mathrm{P}=0.1)$.

\section{Effect of verapamil}

During atrial pacing at a cycle length of $300 \mathrm{msec}$, AF occurred during determination of atrial ERP in $13 \%$ of patients before and after verapamil infusion. In the setting of simultaneous AV pacing, AF occurred during determination of atrial ERP in $87 \%$ and $33 \%$ of patients, before and after verapamil infusion, respectively. Compared with sinus rhythm and atrial pacing, the prevalence of AF was significantly higher during simultaneous AV pacing before verapamil infusion, but not after verapamil infusion (Table 3).

\section{Discussion}

\section{Main Findings}

In this study, atrial pacing at a cycle length of 300 msec with and without simultaneous ventricular pacing allowed assessment of the effects of an acute increase in atrial pressure independent of the effects of an increase in heart rate. An increase in atrial pressure was found to potentiate the shortening of atrial refractoriness that occurs in response to an increase in atrial rate. It also was found to augment heterogeneity in atrial refractoriness, as reflected by the difference in ERPs at the RAA and AS. The occurrence of AF during rapid pacing was greatly enhanced by the increase in atrial pressure that accompanied simultaneous AV pacing. These findings provide evidence suggesting that the phenomenon of mechanoelectrical feedback occurs in the human atrium.

The results of this study demonstrate that the electrophysiologic changes associated with an increase in atrial pressure are influenced by autonomic tone and calcium channel blockade. Autonomic blockade facilitated the maintenance of $\mathrm{AF}$ induced during simultaneous $\mathrm{AV}$ pacing, probably by promoting heterogeneity in atrial refractoriness. On the other hand, verapamil prevented the shortening of atrial refractoriness that was attributable to an elevated atrial pressure and markedly attenuated the propensity for AF.

\section{Susceptibility to Induced AF During Rapid Pacing}

In this study, a strong relationship was found between susceptibility to $\mathrm{AF}$ and right atrial pressure and atrial refractoriness. The prevalence of $\mathrm{AF}$ increased to beyond $90 \%$ as the right atrial pressure increased to levels $>13$ $\mathrm{mmHg}$ and as atrial ERP shortened to values $<140 \mathrm{msec}$. These critical values of atrial pressure and atrial ERP were attained much more often during simultaneous AV pacing than during atrial pacing, which explains why AF was more common during simultaneous AV pacing.

Prior experimental studies demonstrated that shortening of atrial ERP and an increase in atrial pressure independently increase the vulnerability to AF. ${ }^{7}$ Shortening of atrial refractoriness results in a decrease in atrial wavelength, which predisposes to AF. ${ }^{16,17}$ The increase in atrial pressure that occurred during simultaneous $\mathrm{AV}$ pacing in this study potentiated the shortening in atrial refractoriness that occurred during atrial pacing at a cycle length of $300 \mathrm{msec}$ and may have resulted in an increase in the surface area of the atrium, thereby increasing the likelihood of a critical number of wavelets needed for AF to occur. ${ }^{17}$

\section{Heterogeneity of Atrial Refractoriness}

In this study, the difference in atrial ERPs at the RAA and AS was used as a measure of dispersion in atrial refractoriness and was found to be related to the development of sustained AF. This finding is consistent with the results of several experimental studies demonstrating that heterogeneity of atrial refractoriness plays an important role in maintenance of AF. ${ }^{18-20}$

At a constant pacing cycle length of $300 \mathrm{msec}$, the difference in atrial ERPs between the RAA and AS was found to be two to three times greater during simultaneous AV pacing as during atrial pacing. Although the 
TABLE 3

Changes in Atrial Refractory Period Before and After Verapamil

\begin{tabular}{|c|c|c|c|}
\hline & Before Verapamil & After Verapamil & P Value \\
\hline Mean sinus cycle length $(\mathrm{msec})$ & $638 \pm 72$ & $697 \pm 101$ & 0.8 \\
\hline \multicolumn{4}{|l|}{ RAA ERP (msec) } \\
\hline Sinus rhythm & $228 \pm 21^{*}+$ & $231 \pm 24 * t$ & 0.4 \\
\hline Atrial pacing & $175 \pm 12 *$ & $191 \pm 14$ & 0.002 \\
\hline AV pacing & $148 \pm 25$ & $185 \pm 14$ & $<0.001$ \\
\hline \multicolumn{4}{|l|}{ Changes in RAA ERP $(\mathrm{msec}) \dagger$} \\
\hline Atrial pacing & $-53 \pm 28^{*}$ & $-40 \pm 16^{*}$ & 0.02 \\
\hline AV pacing & $-80 \pm 22$ & $-46 \pm 27$ & $<0.001$ \\
\hline \multicolumn{4}{|l|}{ Atrial fibrillation } \\
\hline Sinus rhythm & $00(0 \%)^{*}$ & $00(0 \%)$ & 1.0 \\
\hline Atrial pacing & $2 / 15(13) *$ & $2 / 15(13 \%)$ & 1.0 \\
\hline $\mathrm{AV}$ pacing & $13 / 15(87 \%)$ & $5 / 15(33 \%)$ & 0.02 \\
\hline \multicolumn{4}{|c|}{ Atrial fibrillation, duration $>10 \mathrm{~min}$} \\
\hline Sinus rhythm & $0(0 \%)$ & $0(0 \%)^{*}$ & 1.0 \\
\hline Atrial pacing & $0(0 \%)$ & $0(0 \%)^{*}$ & 1.0 \\
\hline AV pacing & $5 / 13(38 \%)$ & $4 / 5(80 \%)$ & 0.051 \\
\hline
\end{tabular}

Variables are expressed as mean $\pm 1 \mathrm{SD}$.

$* \mathrm{P}<0.05$ compared with $\mathrm{AV}$ pacing; $\$ \mathrm{P}<0.05$ compared with atrial pacing.

$\dagger$ Changes in pressure as compared with sinus rhythm.

$\mathrm{ERP}=$ effective refractory period; RAA = right atrial appendage.

reason for this observation is unclear, it is possible that the increase in atrial pressure that occurred during simultaneous AV pacing resulted in different degrees of stretch at the RAA and AS, or that different parts of the atrium respond differently to a given degree of stretch. ${ }^{21}$

\section{Autonomic Tone}

In this study, autonomic blockade significantly increased the prevalence of sustained AF during rapid pacing. The increased susceptibility to sustained AF was not attributable to a greater degree of shortening of atrial refractoriness at the RAA, but instead to a lesser degree of shortening in atrial refractoriness at the AS, resulting in a greater degree of heterogeneity in atrial refractoriness. In the absence of autonomic blockade, it is possible that the changes in vagal and/or sympathetic tone that occur during rapid pacing

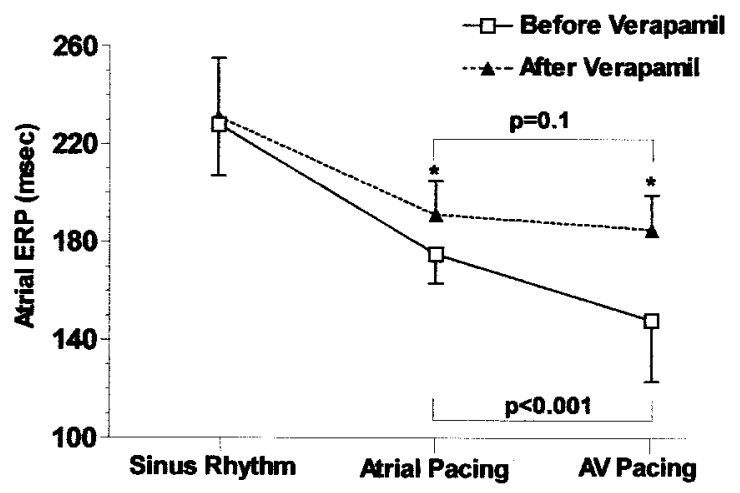

Figure 2. Atrial effective refractory period (ERP) measured at the right atrial appendage during sinus rhythm, during atrial and $A V$ pacing at a cycle length of $300 \mathrm{msec}$ before $(\square)$ and after verapamil $(\mathbf{\Delta})$. Mean values $\pm 1 S D$ and $P$ values are shown. $* P<0.05$ compared with before verapamil. serve to attenuate changes in dispersion of atrial refractoriness. The differential responses of atrial refractoriness to autonomic blockade at different sites in the right atrium suggest that vagal or sympathetic innervation may not be uniform throughout the right atrium..$^{22,23}$

\section{Effect of Verapamil}

Previous experimental studies demonstrated that mechanical stretch results in an increase in intracellular calcium concentration in ventricular myocardium..$^{24-26}$ An increase in intracellular calcium may shorten the

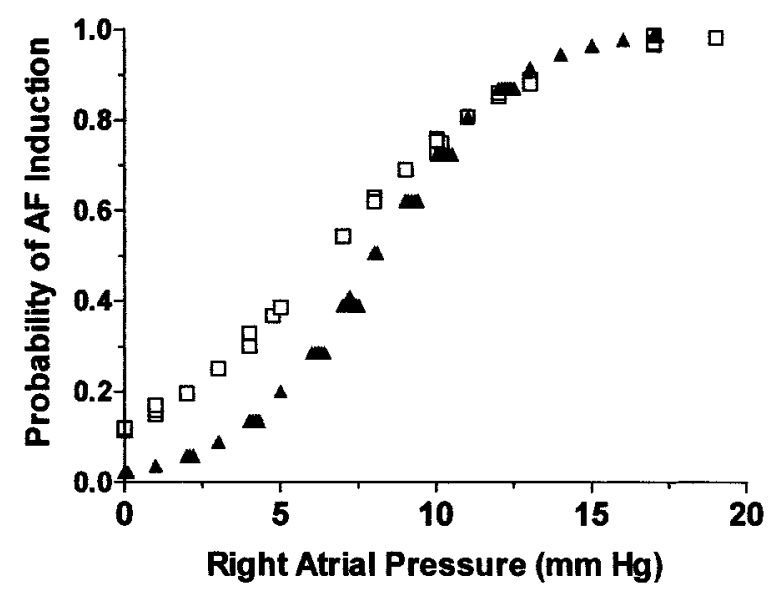

Figure 3. Probability of atrial fibrillation $(A F)$ induction by a single atrial extrastimulus plotted as a function of right atrial pressure in the absence $(\mathbf{\Delta})(r=0.39, P=0.004)$ or presence $(\square)(r=0.35, P=$ 0.02) of autonomic blockade during atrial and simultaneous $A V$ pacing. Datapoints represent the prevalence of $A F$ induced in all patients by a single atrial extrastimulus during measurement of the atrial effective refractory period. 


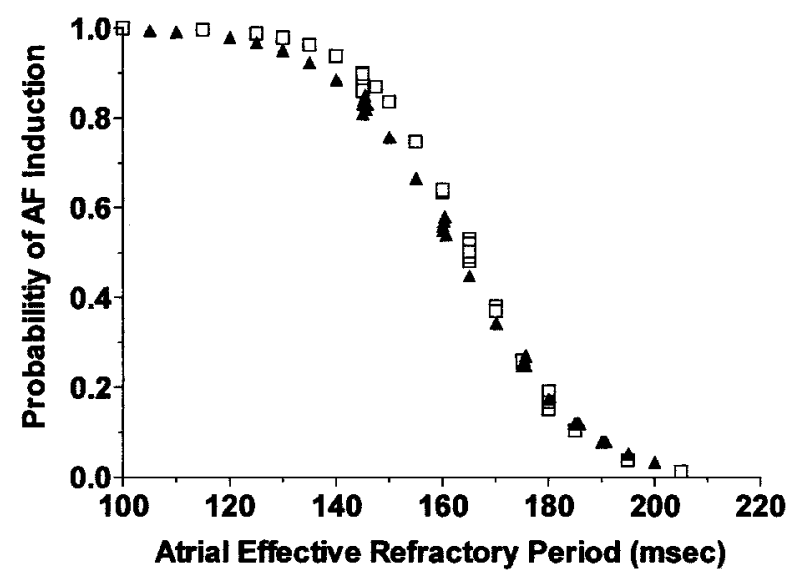

Figure 4. Probability of atrial fibrillation $(A F)$ induction by a single atrial extrastimulus plotted as a function of right atrial appendage effective refractory period in the absence $(\mathbf{\Delta})(r=-0.42, P<0.001)$ or presence $(\square)(r=-0.37, P=0.001)$ of autonomic blockade during atrial and simultaneous $A V$ pacing. Datapoints represent the prevalence of $A F$ induced in all patients by a single atrial extrastimulus during measurement of the atrial effective refractory period.

action potential duration (and shorten refractoriness) by inhibiting transmembrane calcium influx ${ }^{27}$ and activating outward potassium currents such as $\mathrm{I}_{\mathrm{KI}^{28}}$ and $\mathrm{I}_{\mathrm{to} 2}{ }^{28-30}$ Recent experimental studies also demonstrated that verapamil prevents shortening of atrial ERP due to a stretch-induced dilation in atria. ${ }^{31}$ The findings of the present study suggest that a similar dependence of mechanoelectrical feedback on calcium influx may be operative in the atrium.

Prior experimental and clinical studies suggested that the effect of tachycardia on atrial refractoriness may be mediated by tachycardia-induced intracellular calcium overload. ${ }^{32,33}$ Consistent with these findings, verapamil lessened the degree of shortening in atrial refractoriness that occurred in this study as a result of rapid atrial pacing. However, the shortening in atrial refractoriness that occurred as a result of an increase in atrial pressure was completely eliminated by verapamil, suggesting that mechanoelectrical feedback may be more dependent on intracellular calcium loading than is tachycardia-induced shortening of atrial refractoriness. However, because verapamil also blocks potassium channels, ${ }^{34}$ it is possible that some mechanism other than calcium channel blockade was responsible for prevention of atrial electromechanical feedback.

\section{Prior Studies}

Prior experimental studies showed that shortening of refractoriness in response to an increase in pressure occurs in both the atrium ${ }^{5-7}$ and ventricle. ${ }^{35,36}$ In concert with these experimental studies, the results of the present study demonstrate that an increase in atrial pressure is accompanied by a shortening of atrial refractoriness and an increased propensity for AF. However, prior studies in humans reported either no change,, 9 a shortening,,${ }^{10}$ or a prolongation $8,11,12$ in atrial refractoriness during an increase in atrial pressure. The reason for this discrepancy is unclear, but it may be related to differences in the basic drive cycle length used to measure refractoriness ${ }^{37}$ or in the degree of elevation of right atrial pressure. ${ }^{9}$

\section{Limitations}

A limitation of this study is that pacing was performed at only a single cycle length of $300 \mathrm{msec}$. It is possible that the responses to pacing would have been different at longer or shorter pacing cycle lengths. A second limitation is that atrial ERP was measured at only two right atrial sites, yielding at best only a rough estimate of dispersion in atrial refractoriness. Finally, all of the subjects in this study were free of structural heart disease, and the findings in these subjects may not apply to patients who have heart disease.

\section{Conclusion}

The results of this study demonstrate that spontaneous $\mathrm{AV}$ pacing leads to shortening of atrial refractoriness and an enhanced propensity for AF in response to an acute increase in atrial pressure, which may be manifestations of atrial mechanoelectrical feedback in humans. Modulation of the response of atrial refractoriness to an increase in atrial pressure by fluctuations in autonomic tone may play a role in the maintenance of AF. Furthermore, verapamil markedly attenuates the electrophysiologic consequences of an acute rise in atrial pressure, suggesting that they may be mediated by intracellular calcium loading. Although the extent to which mechanoelectrical feedback contributes to the development of clinical episodes of AF is unclear, the findings of this study suggest that an increase in atrial pressure in the setting of a rapid heart rate is likely to increase the susceptibility to tachycardia-induced AF.

\section{References}

1. Cristal N, Peterburg I, Szwarcberg J: Atrial fibrillation developing in the acute phase of myocardial infarction: Prognostic implications. Chest 1976;70:8-11.

2. Manyari DE, Patterson C, Johnson D, Melendez L, Kostuk WJ, Cape RDT: Atrial and ventricular arrhythmias in asymptomatic active elderly subjects: Correlation with left atrial size and left ventricular mass. Am Heart J 1990;119:1069-1076

3. Selzer A, Cohn KE: Natural history of mitral stenosis: A review. Circulation 1972;45:878-890.

4. Solti F, Vecsey T, Kèkesi V, Juhasz-Nagy A: The effect of atrial dilatation on the genesis of atrial arrhythmias. Cardiovasc Res 1989;23:882-886.

5. Nazir SA, Lab MJ: Mechanoelectric feedback and atrial arrhythmias. Cardiovasc Res 1996;32:52-61.

6. Nazir SA, Lab MJ: Mechanoelectric feedback in the atrium of the isolated guinea-pig heart. Cardiovasc Res 1996;32:112-119.

7. Ravelli F, Allessie M: Effects of atrial dilatation on refractory period and vulnerability to atrial fibrillation in isolated Langendorff perfused rabbit heart. Circulation 1997;96:1686-1695.

8. Klein LS, Miles WM, Zipes DP: Effect of atrioventricular interval during pacing or reciprocant tachycardia on atrial size, pressure, and refractory period: Contraction-excitation feedback in human atrium. Circulation 1990;82:60-68. 
9. Calkins H, El-Atassi R, Leon A, Kalbfleisch S, Borganelli M, Langberg J, Morady F: Effect of the atrioventricular relationship on atrial refractoriness in humans. PACE 1992;15:771-778.

10. Calkins H, El-Atassi R, Kalbfleisch S, Langberg J, Morady F: Effects of acute increase in atrial pressure on atrial refractoriness in humans. PACE 1992;15:1674-1680.

11. Chen YJ, Chen SA, Tai CT, Wen ZC, Feng AN, Ding YA, Chang MS: Role of atrial electrophysiology and autonomic nervous system in patients with supraventricular tachycardia and paroxysmal atrial fibrillation. J Am Coll Cardiol 1998;32:732-738.

12. Chen YJ, Tai CT, Chiou CW, Wen ZC, Chan P, Lee SH, Chen SA: Inducibility of atrial fibrillation during atrioventricular pacing with varying intervals: Role of atrial electrophysiology and the autonomic nervous system. J Cardiovasc Electrophysiol 1999;10:15781585 .

13. Jose AD, Taylor RR: Autonomic blockade by propranolol and atropine to study intrinsic myocardial function in man. J Clin Invest 1969;48:2019-2031.

14. Reiter MJ, Shand DG, Aanonsen LA, Wagoner R, McCathy E, Pritchett ELC: Pharmacokinetics of verapamil: Experience with a sustained intravenous infusion regimen. Am J Cardiol 1982;50: 716-721.

15. Daoud EG, Bogun F, Goyal R, Harvey M, Man CK, Strickberger SA, Morady F: Effect of atrial fibrillation on atrial refractoriness in humans. Circulation 1996;94:1600-1606.

16. Moe GK, Rheinboldt WC, Abildskov JA: A computer model of atrial fibrillation. Am Heart J 1964;67:2961-2967.

17. Allessie MA, Rensma PL, Brugada J, Smeets JLRM, Penn O, Kirchhof CJHJ: Pathophysiology of atrial fibrillation. In Zipes DP, Jalife J, eds: Cardiac Electrophysiology: From Cell to Bedside. WB Saunders, Philadelphia, 1990, pp. 548-559.

18. Wang J, Liu L, Feng J, Nattel S: Regional and functional factors determining induction and maintenance of atrial fibrillation in dogs. Am J Physiol 1996;27:H148-H158.

19. Fareh S, Villemaire C, Nattel S: Importance of refractoriness heterogeneity in the enhanced vulnerability to atrial fibrillation induction caused by tachycardia-induced atrial electrical remodeling. Circulation 1998;98:2202-2209.

20. Olgin JE, Sih HJ, Hanish S, Jayachandran V, Wu J, Zheng HQ, Winkle W, Mulholland KG, Zipes DP, Hutchins G: Heterogeneous atrial denervation creates substrate for sustained atrial fibrillation. Circulation 1998;98:2608-2614.

21. Satoh T, Zipes DP: Unequal atrial stretch in dogs increases dispersion of refractoriness conducive to developing atrial fibrillation. J Cardiovasc Electrophysiol 1996;7:833-842.

22. Liu L, Nattel S: Differing sympathetic and vagal effects on atrial fibrillation in dogs: Role of refractoriness heterogeneity. Am J Physiol 1997;273:H805-H816.

23. Warner MR, Zipes DP: Vagal control of myocardial refractoriness. In Levy MN, Schwartz PJ, eds: Vagal Control of the Heart:
Experimental Basic and Clinical Implications. Futura Publishing Co., Armonk, NY, 1994, pp. 261-275.

24. Franz MR: Mechano-electrical feedback in ventricular myocardium. Cardiovasc Res 1996;32:15-24.

25. White E, Le Guennec JY, Nigretto JM, Gannier F, Argibay JA, Garnier D: The effects of increasing cell length on auxotonic contractions, membrane potential and intracellular calcium transients in single guinea-pig ventricular myocytes. Exp Physiol 1993;78:65-78.

26. Lab MJ, Allen DG, Orchard CH: The effects of shortening on myoplasmic calcium concentration and on the action potential in mammalian ventricular muscle. Circ Res 1984;55:825-829.

27. Sun H, Leblanc N, Nattel S: Mechanisms of inactivation of L-type calcium channels in human atrial myocytes. Am J Physiol 1997; 272:H1625-H1635.

28. Di Francesco D, McNaughton DA: The effects of calcium ion outward membrane currents in the cardiac Purkinje fibre. J Physiol (Lond) 1979;289:347-373.

29. Escande D, Coulombe A, Faivre JF, Deroubaix E, Coraboeuf E: Two types of transient outward currents in adult atrial cells. Am J Physiol 1987;252:H142-H148.

30. Shibata EF, Drury T, Refsum H, Aldrete V, Giles W: Contributions of a transient outward current to repolarization in human atrium. Am J Physiol 1989;257:H1773-H1781.

31. Zarse M, Athanatou E, Robert J, Stellbrink C, Hanrath P: Verapamil reverses atrial electrical remodeling due to dilatation in Langendorff-perfused rabbit hearts. (Abstract) J Am Coll Cardiol 2000;I-146A.

32. Daoud EG, Knight BP, Weiss R, Bahu M, Paladino W, Goyal R, Man CK, Strickberger SA, Morady F: Effect of verapamil and procainamide on atrial fibrillation induced electrical remodeling in humans. Circulation 1997;96:1542-1550.

33. Tieleman RG, De Langen CDJ, Van Gelder IC, de Kam PJ, Grandjean J, Bel KJ, Wijffels MCEF, Allessie MA, Crijns HJGM: Verapamil reduces tachycardia-induced electrical remodeling of the atria. Circulation 1997;95:1945-1953.

34. Zhang S, Zhou Z, Gong Q, Makielski JC, January CT: Mechanism of block and identification of the verapamil binding domain to HERG potassium channels. Circ Res 1999;84:989-998.

35. Lerman BB, Burkhoff D, Yue DT, Franz MR, Sagawa K: Mechanoelectrical feedback: Independent role of preload and contractility in modulation of canine ventricular excitability. J Clin Invest 1985;76:1843-1850.

36. Calkins H, Maughan WL, Kass DA, Sagawa K, Levine JH: Electrophysiological effect of volume load in isolated canine hearts. Am J Physiol 1989;256:H1697-706.

37. Reiter MJ, Landers M, Zetelaki Z, Kirchhof CJH, Allessie MA: Electrophysiological effects of acute dilatation in the isolated rabbit heart: Cycle length-dependent effects on ventricular refractoriness and conduction velocity. Circulation 1997;96:4050-4056. 